\title{
Impact of Sr content on dielectric and electrical properties of pulsed laser ablated $\mathrm{SrBi}_{2} \mathrm{Ta}_{2} \mathrm{O}_{9}$ thin films
}

\author{
S. Bhattacharyya, Apurba Laha, and S. B. Krupanidhia) \\ Materials Research Center, Indian Institute of Science, Bangalore-560 012, India
}

\begin{abstract}
The ferroelectric properties of $\mathrm{SrBi}_{2} \mathrm{Ta}_{2} \mathrm{O}_{9}$ thin films grown by laser ablation were investigated as a function of the $\mathrm{Sr}^{+2}$ content in the films. Different target compositions were used to obtain films with different $\mathrm{Sr}^{+2} / \mathrm{Ta}^{+5}$ ratios. The initial composition was according to the stoichiometric composition (1/2), and the $\mathrm{Sr}^{+2} / \mathrm{Ta}^{+5}$ ratio was varied to $0.7 / 2.0$. It was seen that the remanent polarization showed a consistent increase, as the film became more deficient of " $\mathrm{Sr}^{+2}$ " up to a certain extent. Similarly, a decrease in the dielectric constant and the leakage current with the decrease of $\mathrm{Sr}^{+2}$ in the film was observed. The dielectric transition temperature showed an increase with the reduction of $\mathrm{Sr}^{+2}$ content and was seen to approach the bulk value.
\end{abstract}

\section{INTRODUCTION}

There has been an increasing interest in the area of ferroelectric materials owing to their interesting properties that would be useful in memory devices. ${ }^{1,2}$ There are several ongoing research efforts ${ }^{3,4}$ searching for the most suitable ferroelectric material that has reasonably high remanent polarization, low coercive field, and a very high fatigue lifetime to ensure the reliability of a nonvolatile random access memory. There had been an extensive survey on lead based perovskite materials since they were seen to exhibit a very high value of the remanent polarization $\left(35 \mu \mathrm{C} / \mathrm{cm}^{2}\right),{ }^{5,6}$ But they did suffer from severe fatigue problems that caused the remanent polarization to drop down by more than $20 \%$ after repeated read/write operations. ${ }^{7}$ Therefore, a new group of materials was sought for this purpose, and it was seen that the bismuth layered ferroelectric system could provide a fatigue free performance even beyond $10^{12} \mathrm{read} /$ write cycles. $^{8}$ These materials possess a lower coercive field and, being free from lead, they are relatively easier to be processed. The most promising among this group of materials are $\mathrm{SrBi}_{2} \mathrm{Ta}_{2} \mathrm{O}_{9}$ (SBT) and $\mathrm{SrBi}_{2} \mathrm{Nb}_{2} \mathrm{O}_{9}$. There are numerous reports that are mostly related to the optimization of their deposition conditions and characterizations of their various properties, ${ }^{9-11}$ and to understand the underlying mechanism for their fatigue resistance. The effects of the addition of other elements into the various sites of the layered perovskite lattice have also been studied. ${ }^{12,13}$ To improve the ferroelectric properties, efforts have already been made to grow the nonstoichiometric SBT thin films by sol-gel technique as well as metal-organic chemical-vapor deposition technique. ${ }^{14-16}$ It was reported by Noguchi et al. ${ }^{17}$ that SBT thin films of 0.8/2.2/2 (Sr/Bi/Ta) composition showed an improvement in remanent polarization. In the present article, we have studied the effects of "Sr" deficiency in laser ablated $\mathrm{SrBi}_{2} \mathrm{Ta}_{2} \mathrm{O}_{9}$ (SBT) thin films. The impact of the $\mathrm{Sr}(\%)$

\footnotetext{
${ }^{a}$ Author to whom correspondence should be addressed; electronic mail:
} sbk@mrc.iisc.ernet.in content in SBT thin films has been studied in light of their structural properties, and the electrical properties, which included polarization hysteresis, ac conductivity, phase transition, and the dc leakage properties, etc. The reversible and irreversible contributions to the total polarization as a function of $\mathrm{Sr}^{+2}$ content was also estimated in this article in the light of the Rayleigh analysis, which is applicable both for magnetic and ferroelectric materials. ${ }^{18-20}$

\section{EXPERIMENT}

Polycrystalline SBT thin films were grown using a $\mathrm{KrF}$ pulsed excimer laser ablation technique (Lambda Physik, $248 \mathrm{~nm}$ wavelength). A dense single phase SBT ceramic pallet was used as the target. The base pressure was brought down to $2 \times 10^{-5}$ Torr prior to the oxygen incorporation into the deposition chamber. The substrate temperature during deposition was maintained at $400{ }^{\circ} \mathrm{C}$, while oxygen partial pressure was 100 mTorr. The as-grown films were subjected to ex situ annealing at different temperatures from 700 to $800{ }^{\circ} \mathrm{C}$ for $30 \mathrm{~min}$. The films were structurally characterized by means of x-ray diffraction (Scintag, xds2000), and scanning electron microscopy (Cambridge Instruments) was used to study the microstructure, while the composition of the films was determined by energy dispersive $\mathrm{x}$-ray analysis (Oxford Instruments). Gold dots of $800 \AA$ thickness and 500 $\mu \mathrm{m}$ diameter were deposited to form the top electrodes on the films. Ferroelectric hysteresis properties were studied by means of an RT-66A ferroelectric test system (with a test pulse width of $8 \mathrm{~ms}$.). Capacitance-voltage $(C-V)$ characteristics were studied using a Keithley 3330 LCZ meter (coupled with a Keithley 230 voltage source) at frequencies from 1 to $100 \mathrm{kHz}$ with an oscillation amplitude of $50 \mathrm{mV}$. The dielectric and ac electric properties of SBT thin films were investigated using an LCZ meter (model Keithley 3330). Impedance analysis was carried out over a frequency range of $0.1-100 \mathrm{kHz}$ at the temperature range of $30-350{ }^{\circ} \mathrm{C}$. 


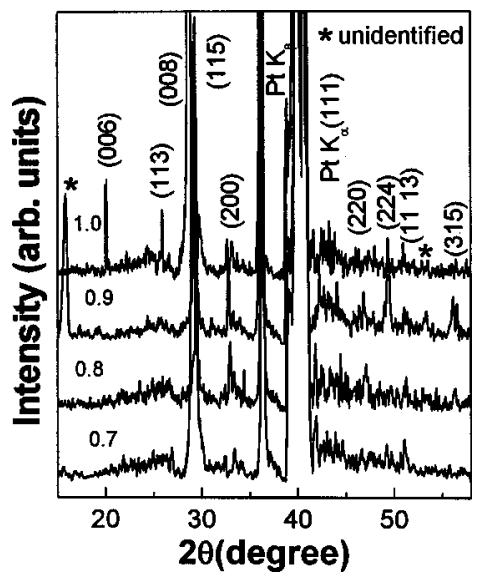

FIG. 1. XRD patterns for various $\mathrm{Sr}$ compositions. The $\mathrm{Sr}$ contents for the samples are mentioned on the respective curves.

\section{STRUCTURE}

Figure 1 shows the x-ray diffraction (XRD) patterns of the $\mathrm{Sr}_{x} \mathrm{Bi}_{2.5} \mathrm{Ta}_{2} \mathrm{O}_{9}$ thin films with " $x$ " varying from 1 to 0.7 . All these films were annealed at a temperature of $750{ }^{\circ} \mathrm{C}$ for half an hour. The XRDs of the samples showed the existence of a polycrystalline phase. However, the degree of polycrystallinity was different for different $\mathrm{Sr}^{+2}$ content. The degree of randomness of orientation was concluded by looking at the ratio of the intensities of the a to c axis peaks. It was observed that the $\mathbf{c}$ to $\mathbf{a}$ axis peak ratio decreased towards the $\mathrm{Sr}^{+2}$ deficient phases, which indicated a more preferred orientation along that (a) direction. One more phenomenon was observed from the (200) peak positions for films with different $\mathrm{Sr}^{+2}$ content. The peak exhibited a shift towards higher angles for lower $\mathrm{Sr}^{+2}$ concentrations. It was already reported that the lowering of the amount of $\mathrm{Sr}^{+2}$ could cause an increase of grain size, and also the bismuth ions would occupy the $\mathrm{Sr}^{+2}$ positions in the $\left(\mathrm{SrTaO}_{6}\right)$ octahedra. ${ }^{21}$ Bismuth ions are smaller in size compared to that of strontium, so that the lattice would contract, which was reflected in the shift of the XRD peaks towards the higher angles in the present case.

\section{COMPOSITION ANALYSIS}

The composition analysis obtained from the EDAX measurements showed that there was indeed a strontium deficiency in the resultant films. There was also a slight bismuth loss during the heat treatment. It was already checked that a target composition with $\mathrm{Sr} / \mathrm{Bi} / \mathrm{Ta}=1 / 2.5 / 2$ could produce a stoichiometric SBT film after the heat treatment. Hence, in the case of $\mathrm{Sr}$ deficient films, the initial composition of the target was chosen as $\mathrm{Sr} / \mathrm{Bi} / \mathrm{Ta}=(1-x) /(2.5+x) / 2.00$. With this initial composition, the films obtained after annealing had a composition of $\mathrm{Sr}_{(1-x)} \mathrm{Bi}_{(2+x)} \mathrm{Ta}_{2} \mathrm{O}_{9}$. The excess bismuth after the annealing gave an indication of the bismuth ions occupying the vacant $\mathrm{Sr}$ sites.

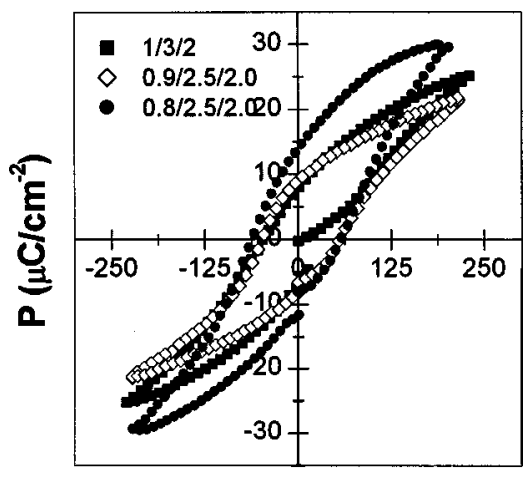

$E(k V / c m)$

FIG. 2. The polarization-electric field hysteresis loops for different nonstoichiometric compounds.

\section{ELECTRICAL PROPERTIES}

\section{A. The polarization hysteresis}

The hysteresis loops for different compositions are shown in Fig. 2. It is evident from the figure that there is an increase in the remanent polarization with the incorporation of $\mathrm{Sr}^{+2}$ vacancies into the lattice (shown in Fig. 3). This is consistent with the earlier reports, ${ }^{17}$ and was explained on the basis of the bismuth ions occupying the vacant $\mathrm{Sr}^{+2}$ sites, because the ionic radii of $\mathrm{Sr}^{+2}$ and $\mathrm{Bi}^{+3}$ are nearly equal. ${ }^{22}$ It is also known that the ionic polarizability of $\mathrm{Bi}^{+3}$ is higher than that of $\mathrm{Sr}^{+2}$, which could make the polarization increase with this replacement. It has been also observed that the grain size of the samples with lesser $\mathrm{Sr}^{+2}$ content is larger than the stoichiometric value. Inside a larger grain, the motion of the domain walls would be relatively easier, since it would come across a lesser number of grain boundaries, resulting in relatively less chances of domain pinning. The domain pinning was explained to be one of the major causes of the decrease of polarization in fatigued ferroelectric samples. ${ }^{23}$

There was a slight increase in the coercive field in the $\mathrm{Sr}^{+2}$-deficient samples. A similar phenomenon was observed for sol-gel derived $\mathrm{Sr}^{+2}$-deficient thin films, ${ }^{17}$ and was attributed to the increased polarizability due to the replacement of $\mathrm{Sr}^{+2}$ ions by the $\mathrm{Bi}^{+3}$ ions. This could make the polarization

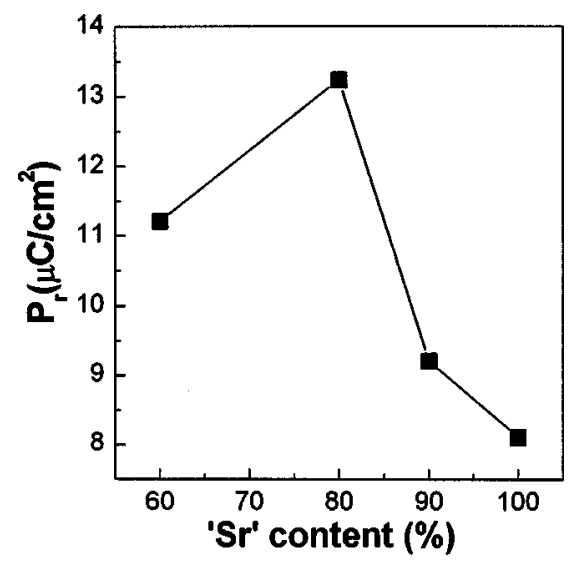

FIG. 3. The remanent polarization $\left(P_{r}\right)$ as a function of $\mathrm{Sr}$ content. 


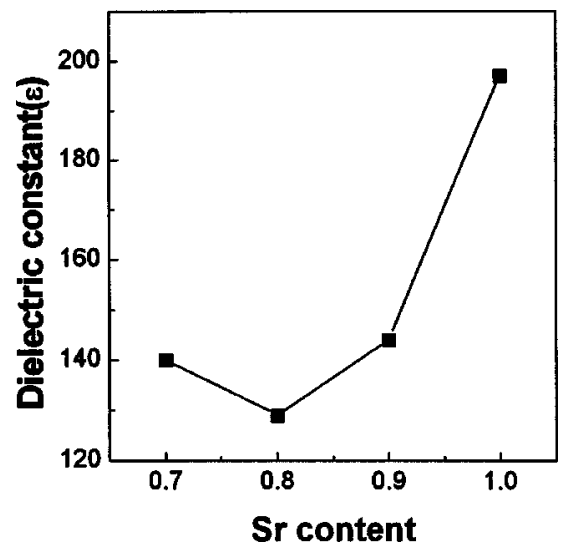

FIG. 4. Dielectric constant $(\epsilon)$ as a function of Sr content.

more stable so that it would not be easily disturbed by the application of external field. The saturation polarization, however, did not show any trend of increase or decrease with the $\mathrm{Sr}^{+2}$ content.

\section{B. The dielectric and ac electrical studies}

Figure 4 shows the change in the dielectric constant with different Sr content. The results were apparently contradictory to the results obtained from the hysteresis measurements. Like the remanent polarization, the dielectric polarization should also have increased. There could be two explanations for this, one being the increase in the porosity in the structure in the film due to the increase of grain size, and hence the effective density of dipole moment was reduced. The other explanation would be the difference between the inherent and the induced polarizations. The remanent polarization is considered to be the inbuilt deformation of the unit cell, while the dielectric constant corresponds to the induced polarization, which involves small displacements of ions about their mean positions (in ferroelectric materials, the positions of the ions are such that the system is spontaneously polarized). Such displacements could be induced by the application of a small signal external field. The higher remanent (or spontaneous) polarization would correspond to a highly deformed perovskite cell, and very little further distortion may be allowed by the application of an external field. However, there was an anomaly observed below a $\mathrm{Sr}^{+2}$ percentage of $80 \%$. The polarization started to decrease after this value of the $\mathrm{Sr}^{+2}$ percentage. The dielectric constant started increasing beyond this composition. To distinguish between the small signal and large signal responses, we have performed the Rayleigh analysis on our samples by varying the ac signal amplitude and observing its effect. It was known that the large signal response of a ferroelectric film consists of a reversible polarization, and the rest is irreversible polarization, which is responsible for its hysteresis loop. ${ }^{24}$ On the other hand, it is also known that the small signal response, which is supposed to be reversible, also exhibits some amount of irreversibility, ${ }^{19}$ and this irreversibility increases with the increase of the signal amplitude. This irreversibility is believed to arise from the domain wall pinning at the defect sites (the dielectric constant comes due to

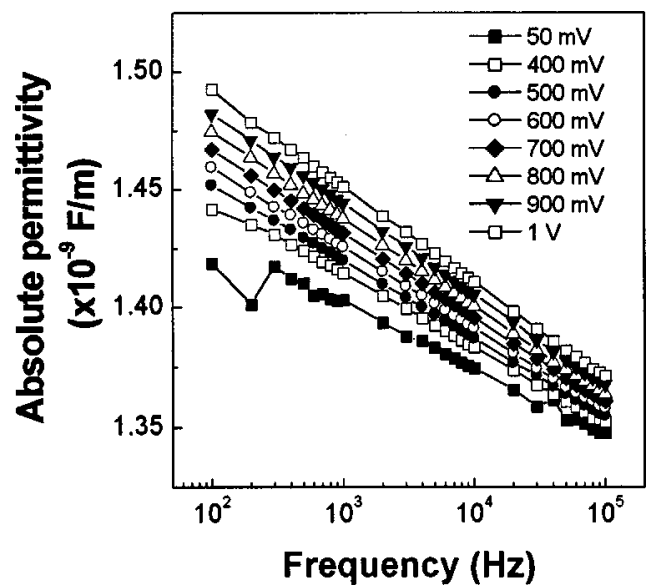

FIG. 5. Absolute permittivity in the Rayleigh plot for $\mathrm{Sr}_{0.8} \mathrm{Bi}_{2.5} \mathrm{Ta}_{2} \mathrm{O}_{9}$ thin films.

the domain wall contribution at lower fields). The trap centers would be separated by small energy barriers, which could be overcome with a little higher signal field. This would lead to an irreversibility, and hence a nonlinearity in the dielectric constant. The magnitude of the dielectric constant is known to vary with the signal amplitude according to the following relation:

$$
\epsilon=\epsilon_{\text {initial }}+\alpha E_{0},
$$

where $\epsilon_{\text {initial }}$ is the dielectric constant at very low signal voltage, and $E_{0}$ is the oscillation field produced by the signal voltage, for a thin film, which might be significantly high. The parameter " $\alpha$ " is known as the Rayleigh parameter.

According to Rayleigh's theory, the frequency dispersion of the dielectric constant would be as follows:

$$
\epsilon(\omega)=\epsilon(0)-k \log \omega .
$$

This is what is known as the domain-wall relaxation.

The result of this kind of measurement for a typical sample (with a $\mathrm{Sr}$ content of 0.8) is displayed in Fig. 5. The dielectric constant followed Rayleigh type dispersion quite accurately; the behavior was more evident at higher signal amplitudes. This was most probably due to the improved sensitivity of the measuring instrument at higher signal voltages. The signal amplitude $\left(E_{0}\right)$ dependence of the dielectric constant is also shown in Fig. 6 for the same film. The other films (with different $\mathrm{Sr}$ content) also exhibited a similar type of behavior. The variation of the parameter ' $\alpha$ ' is shown in the inset of Fig. 6. It was seen that the parameter $\alpha$ decreased with the decrease of the Sr percentage in the sample. This re-established the validity of Rayleigh's law in the case of ferroelectric materials also. Kronmüller ${ }^{18}$ proved that, in case of magnetic materials, the value of the Rayleigh coefficient is inversely proportional to the density of dislocations. Waser et $a .^{25}$ had shown that this relationship could be applied to doped $\mathrm{BaTiO}_{3}$ samples. In our samples, it could also be assumed that the increased degree of Sr deficiency led to the formation of more defect sites, and henceforth $\alpha$ was decreased. It was reported that the $\mathrm{Pb}^{+3}$ impurities in $\mathrm{Pb}^{+2}$ sites in piezoelectric transducer could act as the pinning centers for the holes. ${ }^{26}$ Consequently, we could assume that the 


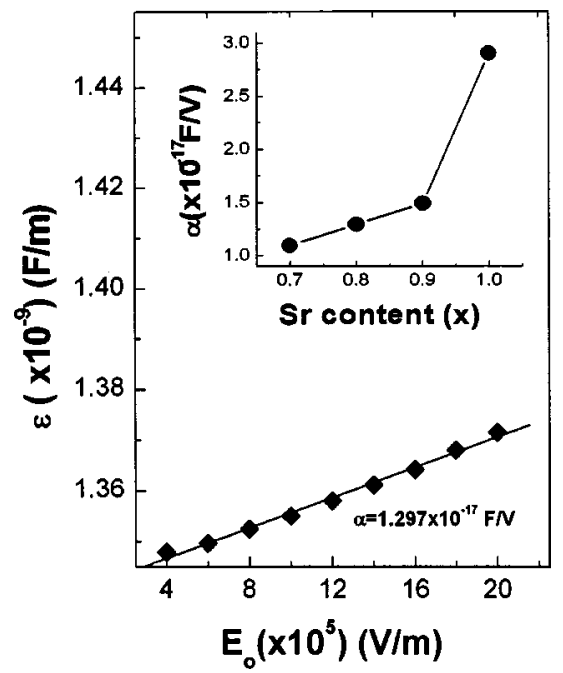

FIG. 6. The absolute permittivity of a typical nonstoichiometric composition $\left(\mathrm{Sr}_{0.8} \mathrm{Bi}_{2.5} \mathrm{Ta}_{2} \mathrm{O}_{9}\right)$ as a function of the ac oscillation field amplitude. The Rayleigh constant is shown for different $\mathrm{Sr}$ content in the inset.

$\mathrm{Bi}^{+3}$ ions in the Sr-deficient SBT samples would also behave in the same way. The domain walls would also attract the holes, because near the domain walls there would be unbalanced (even though bound) polarization charges, which could attract free charge carriers. In effect, the domain wall boundary would be trapped due to the presence of the $\mathrm{Bi}^{+3}$ centers. This might be the reason for the lowering of the dielectric constant in the Sr-deficient samples, because it was already mentioned that in Sr-deficient compositions, the vacant $\mathrm{Sr}^{+2}$ sites would be replaced by the $\mathrm{Bi}^{+3}$ ions

\section{C. ac conductivity}

The ac conductivity for three compositions as a function of frequency is shown in Fig. 7. It was seen that, like the other thin films of layered perovskite ferroelectric materials, ${ }^{11}$ the conductivity showed a power law dependence on the frequency of the driving ac field, indicating an interacting system of charge carriers dominating the ac conduction. The exponent of this power law was the same for all

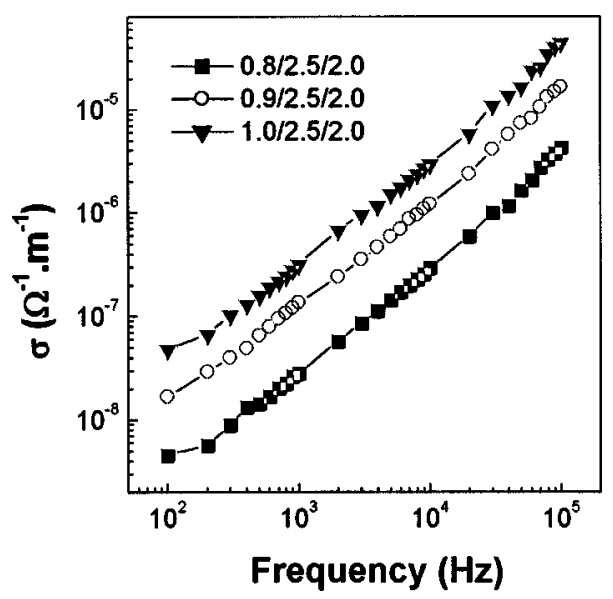

FIG. 7. The ac conductivity plot for different nonstoichiometric compositions.

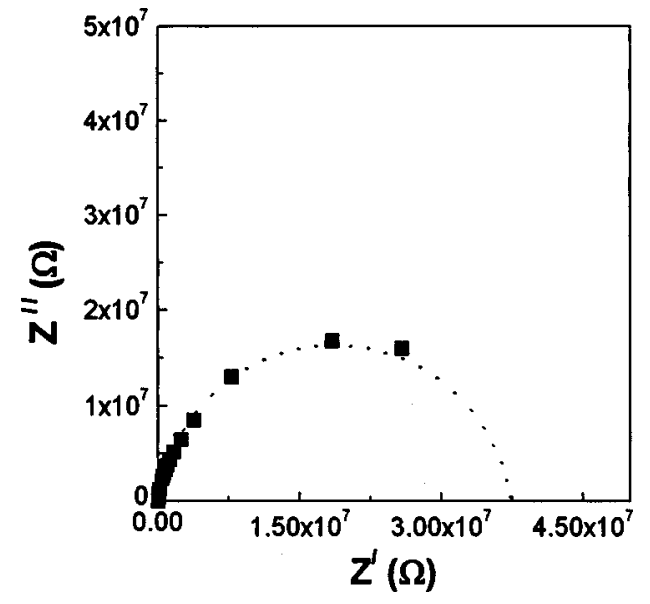

FIG. 8. The Cole-Cole plot of a typical composition with $\mathrm{Sr}$ content $=0.8$.

the compositions of the samples studied. This meant that the change in the Sr content did not result in much change in the interaction parameter. The conductivity of the sample was found to come down as the concentration of the strontium ions was reduced, even though the frequency dependence of the ac conductivity was still the same. This might have been due to the compensation of the $p$-type charge carriers ${ }^{27}$ due to the $\mathrm{Bi}^{+3}$ doping (which would act as donor impurities). It is known ${ }^{28}$ that the bismuth ions after occupying the $\mathrm{Sr}^{+2}$ sites remain in the triply ionized state, so that they could contribute the excess (donor) electrons.

The activation energies were close to that of the oxygen vacancies, indicating that there was a significant contribution from oxygen vacancies in the ac conduction through the sample, particularly at low frequencies. But as a whole, the net conduction phenomenon was a result of the interaction between the charge carriers, and the sample behaved like a Curie-von Scwideler type element, which was evident from the Cole-Cole plots of their ac impedance in the complex plot (shown in Fig. 8).

\section{The dielectric phase transition}

The ferroparaelectric phase transition curves have been plotted in Figs. 9(a) and 9(b), respectively. It was seen that the phase transition temperature increased by about $150{ }^{\circ} \mathrm{C}$ with a change of Sr percentage by $20 \%$. This was also observed earlier by different workgroups, ${ }^{14}$ and was attributed to the increase in the stability of ferroelectric phase due to the bismuth doping. The increased coercive field with increasing Sr deficiency also confirmed this. But it should also be mentioned that the transition temperatures were less than the reported values for the bulk SBT $\left(350^{\circ} \mathrm{C}\right) .{ }^{29}$ Particularly, at a stoichiometric concentration of the strontium, the transition temperature was found to be far below the bulk value $\left(180-200^{\circ} \mathrm{C}\right)$, and it was found to increase with the annealing of the films, which was clearly a result of increase in the grain size. Therefore, the observed increase of the phase transition temperature with reduced strontium content might have been, at least for a very less percentage of $\mathrm{Sr}^{+2}$ vacancies, a consequence of the increase of the grain size due to the incorporation of this defect. It is already known that the 

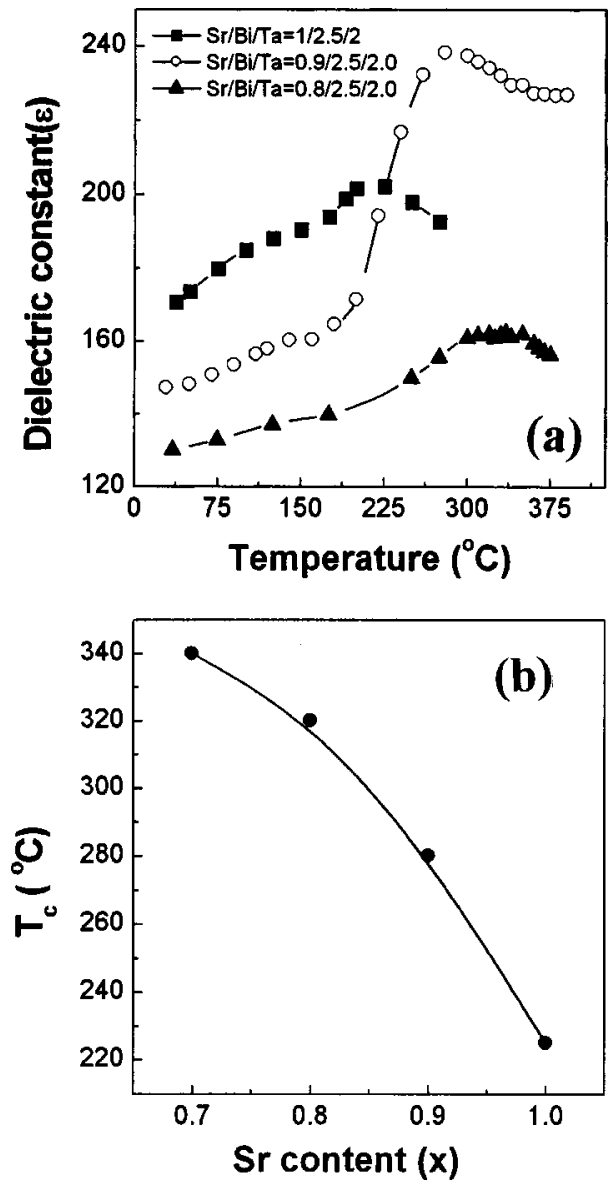

FIG. 9. (a) Dielectric phase transition for three compositions. (b) The transition temperature as a function of the $\mathrm{Sr}$ content.

transition temperature varies very abruptly with grain size for ultrafine grained samples. ${ }^{30}$ For grains of sizes less than a critical value, the transition temperature is usually lesser than the bulk transition temperature and the Curie temperature increases with increasing grain size. In principle, it should asymptotically approach the $T_{c}$ of bulk. However, in our case, as the doping density increased, the system could not

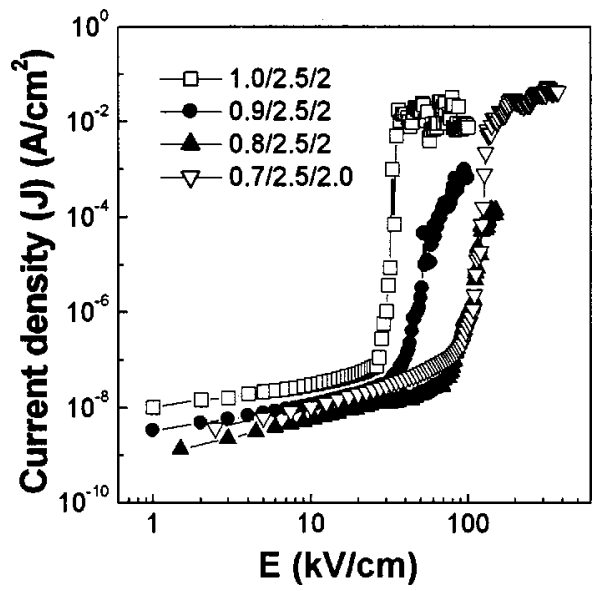

FIG. 10. de leakage current plots for different Sr contents.

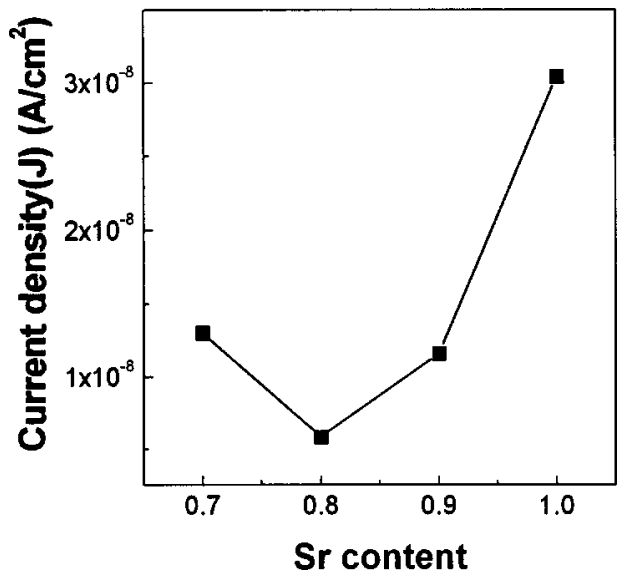

FIG. 11. The variation of the leakage current density as a function of $\mathrm{Sr}$ content at an electric field of $10 \mathrm{kV} / \mathrm{cm}$.

be considered to be just a perturbation to the stoichiometric SBT, rather, it would behave as a completely different system.

\section{E. The dc electrical properties}

The dc current voltage characteristics of the films with varying $\mathrm{Sr}^{+2}$ content are shown in Fig. 10. We have already demonstrated the existence of space charge controlled conduction in SBT films to govern the nonlinear conduction process in the films. ${ }^{31}$ It was seen that the current was a monotonically decreasing function of the percentage of $\mathrm{Sr}$ up to a certain level (80\%). Following this, an increase in the current was observed. The trap filled limit, however, was a steadily increasing function of the $\mathrm{Sr}$ deficiency. The variation of the current density in the linear region $(10 \mathrm{kV} / \mathrm{cm})$ as a function of $\mathrm{Sr}$ content has been separately shown in Fig. 11. The decrease in the leakage current could be attributed to the compensation of the $p$-type charge carriers as explained in the preceding section. After a certain percentage of $\mathrm{Sr}$, when the compensation would have been completed, the film showed a tendency to behave as $n$-type material, and the current started to increase as the $\mathrm{Sr}$ content decreased. From the trap filled limit, the trap density was calculated as a function of the Sr content of the film and is shown in Fig. 12, and

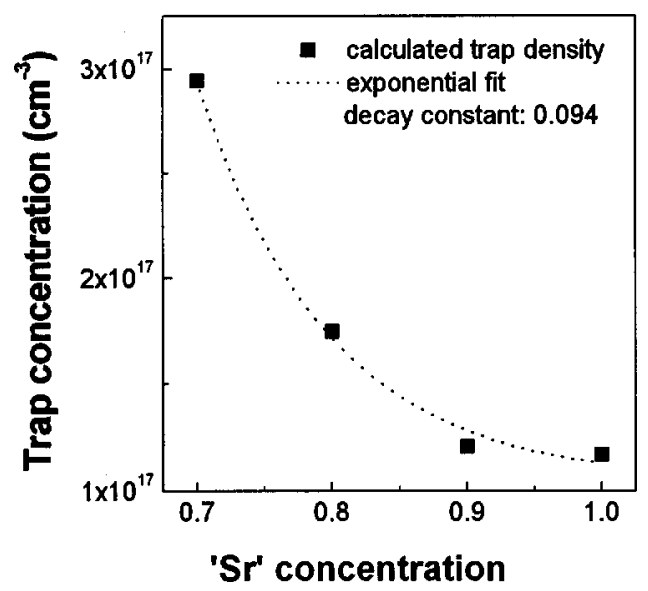

FIG. 12. The trap concentration as a function of $\mathrm{Sr}$ content. 
it was seen to increase with the decrease of the $\mathrm{Sr}$ percentage. This was, however, expected since the incorporation of one $\mathrm{Sr}$ deficiency would introduce more defect states inside the sample, and would also increase the number of traps. However, an interesting observation was that the increase of the trap density was not a linear function of the $\mathrm{Sr}$ deficiency; instead it increased exponentially with the amount of the Sr deficiency. This indicated that the Sr deficiencies alone were not the cause of the increase of the charge traps, but they might have also influenced other atoms/ions which resulted in the formation of more traps than the number of the Sr deficiencies introduced.

\section{CONCLUSIONS}

In conclusion to this section, it may be mentioned that the Sr-deficient compositions exhibited relatively superior ferroelectric and leakage properties over the stoichiometric composition. However, this trend showed an improvement of properties up to a $\mathrm{Sr}$ content of 0.8 , whereas there was a decrease in the ferroelectricity beyond this. Therefore, the nonstoichiometric $\mathrm{Sr}_{0.8} \mathrm{Bi}_{2.5} \mathrm{Ta}_{2} \mathrm{O}_{9}$ composition was the optimum composition for NVRAM applications. The dc leakage also showed a decrease up to $x=0.8$, and then started increasing. The trap concentration was consistently increasing with the $\mathrm{Sr}$ content, due to increase in the disorder in the lattice. The increase in trap concentration was also reflected in the decrease in the Raleigh constant (which would be inversely related with the number of domain pinning centers) with the incorporation of Sr deficiencies.

\footnotetext{
${ }^{1}$ M. E. Scott, C. A. Paz de Araujo, and L. D. McMillan, U. S. Patent No. 9310150, 1993.

${ }^{2}$ Ferroelectric Thin Films V, edited by S. B. Desu, R. Ramesh, B. A. Tuttle, R. E. Jones, and I. K. Yoo, MRS Symposia Proceedings No. 433, San Francisco, April 7-12 (Materials Research Society, Pittsburgh. 1996).

${ }^{3}$ S. D. Bu, B. H. Park, B. S. Kang, T. W. Noth, and W. Jo, Appl. Phys. Lett. 75, 1155 (1999).
}

${ }^{4}$ Y. Nakao, T. Nakamura, A. Kamisawa, H. Takasu, N. Soyama, T. Atsuki, and K. Ogi, Jpn. J. Appl. Phys., Part 1 33, 5265 (1994).

${ }^{5}$ C. J. Kim, D. S. Yoon, J. S. Lee, C. G. Choi, W. J. Lee, and K. No, J. Appl. Phys. 76, 7478 (1994).

${ }^{6}$ Xin-Shan Li, Tsunehisa Tanaka, and Yoshihiko Suzuki, Thin Solid Films 375, 91 (2000).

${ }^{7}$ T. Mihara, H. Watanabe, and C. A. Araujo, Jpn. J. Appl. Phys., Part 133 , 3996 (1994)

${ }^{8}$ J. F. Scott, M. C. Scott, C. A. Paz de Araujo, J. D. Cuchiaro, and L. D. McMillan, Nature (London) 374, 627 (1995).

${ }^{9}$ K. Amanuma, T. Hase, and Y. Miyasaka, Appl. Phys. Lett. 66, 221 (1995).

${ }^{10}$ S. B. Desu and D. P. Vijay, Mater. Sci. Eng., B 32, 83 (1995).

${ }^{11}$ S. Bhattacharyya, S. S. N. Bharadwaja, and S. B. Krupanidhi, Appl. Phys. Lett. 75, 2656 (1999).

${ }^{12}$ W. J. Lee, I. K. You, II-Suk Yang, B. G. Yu, and K. I. Cho, Jpn. J. Appl. Phys., Part 1 39, 5469 (2000).

${ }^{13}$ S. Y. Chen and V. C. Lee, J. Appl. Phys. 87, 8024 (2000).

${ }^{14}$ Y. Noguchi, M. Miyayama, and T. Kudo, J. Appl. Phys. 88, 2146 (2000).

${ }^{15}$ N. Nukaga, M. Mitsuya, T. Suzuki, Y. Nishi, M. Fujimoto, and H. Funakubo, Jpn. J. Appl. Phys., Part 1 49, 5592 (2001).

${ }^{16}$ B. Chendrix et al., Mater. Res. Soc. Symp. Proc. 541, 275 (1999).

${ }^{17}$ T. Noguchi, T. Hase, and Y. Miyasaka, Jpn. J. Appl. Phys., Part 1 35, 4900 (1996).

${ }^{18}$ H. Kronmüller, Z. Angew. Phys. 30, 9 (1970).

${ }^{19}$ D. Damjanovic and N. Setter, J. Appl. Phys. 82, 1788 (1997).

${ }^{20}$ D. Bolten, O. Lohse, M. Grossmann, and R. Waser, Ferroelectrics 221, 251 (2000).

${ }^{21}$ Y. Shimakawa, Y. Kubu, Y. Nakagawa, T. Kamiyama, H. Asano, and F. Izumi, Appl. Phys. Lett. 74, 1904 (1999).

${ }^{22}$ K. Miura and M. Tanaka, Jpn. J. Appl. Phys., Part 1 37, 2554 (1998).

${ }^{23}$ D. Dimos, H. N. Al-Shareef, W. L. Warren, and B. A. Tuttle, J. Appl. Phys. 80, 1682 (1996).

${ }^{24}$ R. Waser, Mater. Res. Soc. Symp. Proc. 596, 301 (2000).

${ }^{25}$ D. Bolten, U. Böttger, T. Schneller, M. Grossmann, O. Lohse, and R. Waser, Appl. Phys. Lett. 77, 3830 (2000).

${ }^{26}$ J. Robertson, W. L. Warren, B. A. Tuttle, D. Dimos, and D. M. Smyth, Appl. Phys. Lett. 63, 1519 (1993).

${ }^{27}$ C. M. Palanduz and D. M. Smyth, J. Eur. Ceram. Soc. 19, 73 (1999).

${ }^{28}$ Huei-Mei Tsai, Pang Lin, Tseung, and Yuen Tseng, J. Appl. Phys. 85, 1095 (1999).

${ }^{29}$ E. C. Subbarao, J. Phys. Chem. Solids 23, 665 (1962).

${ }^{30}$ R. E. Newnham and S. T. McKinstry, Integr. Ferroelectr. 20, 1 (1998).

${ }^{31}$ S. Bhattacharyya, A. Laha, and S. B. Krupanidhi, J. Appl. Phys. 91, 4543 (2002). 